\begin{tabular}{|c|c|c|c|c|c|}
\hline Cuadernos I. Geográfica & 16 & $1-2$ & $123-138$ & Logroño & 1990 \\
\hline
\end{tabular}

\title{
PROCESOS FLUVIALES EN LECHOS CON MATERIALES GRUESOS
}

\author{
Francisca SEGURA BELTRAN*
}

\begin{abstract}
RESUMEN.-LOs mecanismos de transporte de sedimentos gruesos han sido poco investigados. La existencia de una lámina de erosión (scour) durante las crecidas, asi como la deposición de una capa más grosera en superficle (pavement), son dos caracteristicas comunes a la mayoria de los rios con este tipo de sedimentos. El grueso tamaño de los clastos superficiales ejerce una fuerte rugosidad sobre el flujo. Por ello, los cálculos de velocidad media pueden hacerse con fórmulas que estimen la rugosidad a partir del calibre de los materiales.
\end{abstract}

ABSTRACT.-Fluvial processes in gravel-bed rivers. The bedload transport processes in gravel-bed rivers are not very known. The two most frequent features that characterize these rivers are the presence of a scour layer and the pavement. The coarse surface layer causes a great resistance to flow in gravel-bed rivers. So, flow resitance equations for computing average velocity can be applied.

Palabras clave: Capa de erosión, pavimento, rugosidad, velocidad media. Key words: Scour layer, pavement, flow resistance, average velocity.

Los procesos fluviales en cauces de materiales groseros - bloques, cantos y gravas-, han merecido menor atención que en canales con depósitos finos. Los mecanismos de transporte, la composición de la carga de fondo, la lámina de erosión (scour) o la rugosidad y su influencia sobre el flujo plantean problemas de muestreo, de ubicación de los puntos de observación, de diseño de trampas, etc. Ante estas dificultades muchos especialistas han optado por investigar desde supuestos teóricos y pocos trabajos plantean la via experimental.

*Departamento de Geografia. Universitat de Valencla. 
Las ramblas mediterráneas que circulan por áreas calcáreas, ofrecen un tipo excelente de cauce con materiales gruesos. Su lecho está constituido por barras y canales entrelazados y sus sedimentos son una mezcla heterogénea de diferentes calibres. La discontinuidad espacial y temporal de la circulación hídrica -debido al carácter local de las precipitaciones y a las pérdidas de transmisión- contribuyen a que el transporte de sedimentos no sea continuo. Por ello, los materiales están muy mal clasificados y el tamaño de la capa superficial, más grosera, apenas disminuye con la distancia de transporte (SEgurA, 1990). Sin embargo, muchos aspectos del trasporte de materiales en este tipo de ríos siguen siendo prácticamente desconocidos.

Por ello, el presente trabajo trata de dilucidar tres temas en las ramblas: la capa de scour, la influencia de la rugosidad del lecho sobre el flujo y la existencia del armour/pavement.

\section{Metodología}

Para analizar los temas señalados anteriormente, se ha escogido la Rambla de Cervera. Esta rambla recorre un conjunto de fosas y horsts en la zona de contacto entre la Cordillera Ibérica y las Cordilleras Costero Catalanas (SEGURA. 1984, 1986, 1990). El trabajo de campo se realizó entre el 31 de Marzo y el 5 de Mayo de 1989, durante y después de una pequeña crecida acontecida entre marzo y abril. La sección transversal elegida tiene una anchura aproximada de unos $100 \mathrm{~m}$., y en ella se puede distinguir una barra central y varios canales. En uno de ellos, de unos $5 \mathrm{~m}$. de anchura, situado entre la barra central y la margen izquierda, se realizaron las mediciones de las variables utilizadas en los diferentes apartads de este trabajo.

A su vez, la sección del cauce escogido, se desdobla en dos pequeños canales, de escasa profundidad (Fig. $\mathrm{n}^{\circ} 1 \mathrm{~B}$ ). El principal, más profundo, alcanza los 15.5 $\mathrm{cm}$. de calado mientras que el secundario sólo tiene 10.5. En ellos se han medido además otras variables como el calibre de los materiales, la pendiente, y la velocidad de flujo.

En cada uno de los microcanales se han recogido sedimentos, para las que se ha definido diferentes percentiles $\left(D_{n}\right)$. El muestreo, presentaba algunos problemas metodológicos ya que el grueso calibre de los sedimentos superficiales hacian necesario un mestreo areal, mientras las particulas más finas de la capa subyacente requerian un muestreo a peso. Ante el dilema, se optó por muestrear a peso, con el fin de recoger adecuadamente la fracción fina del sedimento.

Por lo que se refiere a la pendiente, LEOPOLD et al. (1964) sugieren que deberían medirse a lo largo de una distancia que sea 10 veces superior a la anchura del canal, que evite distorsiones puntuales. Ello obliga a realizar diversas secciones transversales para obtener la profundidad media lo que, a su vez, hace más prolija la recogida de muestras. Otra opción alternativa es medir la pendiente en un tramo más corto: se obvian las dificultades de muestreo, pero 


\section{PROCESOS FLUVIALES}

se corre el peligro de escoger algún punto con características muy especiales (pool, riffle, etc). En el presente trabajo se optó por la segunda alternativa $(10 \mathrm{~m}$. de longitud) ya que el muestreo se hizo a lo largo de una sección transversal (Tabla 1). La velocidad se evaluó con tres pruebas de flotadores en cada canal. Los resultados fueron muy similares en todos los casos (Tabla 1).

TABLA 1

Características fisicas de los canales

\begin{tabular}{lcccccc}
\hline & Pendiente & Profundidad(m)Velocidad(m/s) & $D_{s o}(m)$ & $D_{s 4}(m)$ & $D_{s o}(\mathrm{~m})$ \\
\hline CANAL PRINCIPAL & 0.0255 & 0.155 & 0.86 & 0.0295 & 0.050 & 0.052 \\
CANAL SECUDARIO & 0.0240 & 0.105 & 0.32 & 0.0260 & 0.051 & 0.056 \\
\hline
\end{tabular}

Para cada uno de los temas analizados se utilizaron diferentes variables. Así, el estudio de la capa de scour se efectuó a partir de los perfiles transversales de la sección durante y después de la crecida (Fig. $n^{2} 1 \mathrm{~A}$ ). Los cálculos de velocidad se hicieron con los datos de la pendiente, del calado y del calibre de los sedimentos obtenidos en cada canal (Fig. $n^{\circ} 1$ B y cuadro $n^{\circ} 1$ ) durante la crecida. Por último, para la descripción de los procesos de pavimentación se recogieron, a lo largo de la sección, cuatro muestras en superficie y otras cuatro en los mismos puntos, pero a diferente profundidad, cuando ya había cesado el flujo (Fig. $n^{\circ} 1 \mathrm{C}$ ).

\section{La lámina de erosión (Scour)}

Como es sabido durante las crecidas fluviales se produce, en mayor o menor grado, una fase de erosión y otra de relleno del lecho (LEOPOLD, WOLMAN y MILLER, 1964). Los sedimentos removidos forman la capa o lámina erosiva (scour) cuyas características -espesor, composición, modalidad y distancia de transporte, etc- tienen una enorme importancia para la comprensión de los procesos de transporte de la carga de fondo (bedload). Para analizarla se han diseñado diversos experimentos, pero existe una cierta controversia sobre los resultados obtenidos. Si en medios áridos esta capa alcanza potencias de varios decímetros (SCHICK, LEKACH y HASSAN, 1987), algunos autores sugieren que es poco profunda en corrientes perennes (REID, FROSTICK y LAYMAN 1985). En las ramblas puede alcanzar espesores de más de $30 \mathrm{~cm}$., aunque para los mismos episodios de crecida, en canales con materiales finos, esta capa tiene importancia (SANJAUME, et al., in fieri). El estado actual de la investigación sugiere que la naturaleza y la movilidad de esta lámina no sólo es función del tipo de crecida, sino también de la cohesión de los materiales del lecho.

Los perfiles de la sección transversal en la Rambla de Cervera (Figura nํ 1 A) revelan la existencia de una capa de erosión cuyo espesor oscial entre 3 y 9 cm., según su ubicación en el canal. Los máximos cambios acontecen en el canal 
principal, por cuanto alli se produce mayor remoción y también la regularización del perfil. En otras palabras, la movilización de sedimentos no es uniforme en toda la sección transversal: la ratio de carga de fondo difiere de la parte central a las márgenes del cauce (REID, Frostich y LAYMAN, 1985).

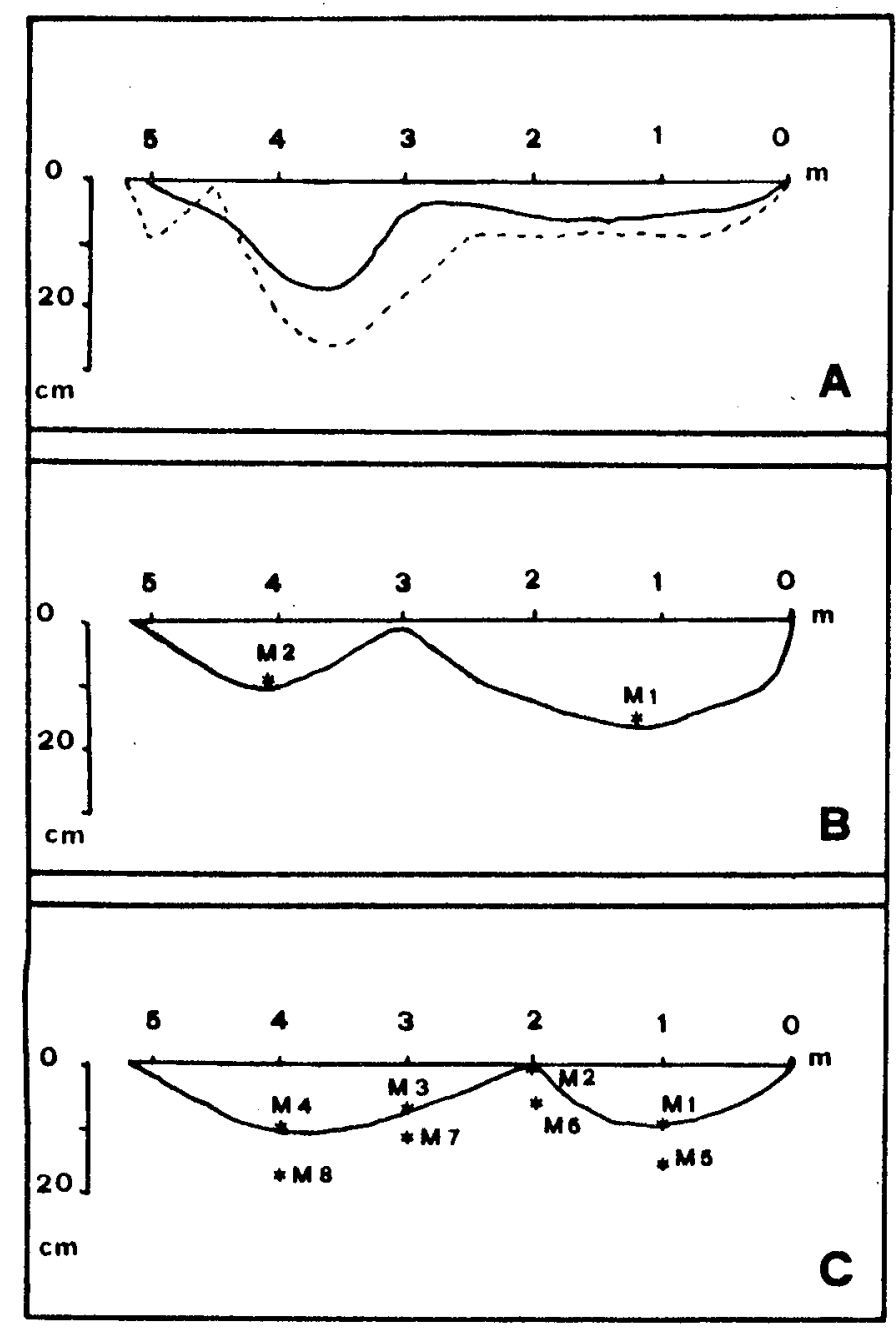

Fig. 1: A) Sección transversal donde se aprecla la lámına de scour. Con trazo discontinuo está señalado el perfll durante la crecida y con linea continua el mismo perfll cuando había cesado el flujo. B) Sección transversal donde se midió la velocidad, con la ubicación de las muestras recogidas. C) Corte transversal con la localización de las muestras correspondientes al pavimento y al subpavimento.

\section{Estimación de la velocidad de flujo en canales no aforados}

La estimación de la velocidad y del caudal en lugares no aforados es tarea árdua. Las dificultades son mayores si cabe en lechos con materiales gruesos, porque además de la anchura, profundidad y pendiente, es necesario estimar la rugosidad sobre el flujo. 
Para solucionar este problema se han utilizado diversas fórmulas empíricas. Una de las más habituales es la de MANNING. En ella el valor $n$, se obtiene generalmente a partir de tablas -la más usada es la de CHOW (1954)- que describen cualitativamente los diferentes tipos de canales, asignándoles un valor especifico. Sin embargo, la validez de esta fórmula ha sido discutida en lechos con materiales gruesos, razón por la cual diferentes autores han propuesto modificaciones a la fórmula de MANNING o bien han sugerido otras nuevas (KeULEGAN, 1938; LIMERINOS, 1970; COWAN, 1954; LACEY, 1946). Investigaciones posteriores (HEY, 1979; BRAY, 1979, 1982) han intentado mejorar los resultados modificando los coeficientes propuestos por aquellos. Su originalidad estriba en calibrar la rugosidad a partir del diámetro de los sedimentos del lecho, superando la dificultad de trabajar con coeficientes elegidos de manera arbitraria.

El objetivo del presente apartado es comprobar la bondad de algunas de estas fórmulas utilizando los datos medidos durante una crecida registrada en la Rambla de Cervera. Para ello siguiendo la metodologia propuesta por BRAY $(1979,1982)$ se han contrastado las fórmulas de MANNING-LIMERINOS, KEULEGAN, LACEY y también la de LEOPOLD et al. (1964):

$$
\begin{array}{ll}
\mathrm{V}=\frac{\mathrm{d}^{2 / 3} \mathrm{~S}^{1 / 2}}{0,113 \mathrm{~d}^{1 / 6}} & (1=\text { MANNING-LIMERINOS }) \\
\mathrm{V}=\left(\mathrm{g} \mathrm{d} \mathrm{s}^{1 / 2}\left(6.25+5.75 \log \left(\frac{\mathrm{d}}{\mathrm{D}_{84}}\right)\right)\right. & (2=\text { KEULEGAN }) \\
V=10.8 \mathrm{~d}^{2 / 3} \mathrm{~S}^{1 / 3} & (3=\text { LACEY }) \\
V=\left(\mathrm{g} \mathrm{d} \mathrm{s}^{1 / 2}\left(2.83+5.75 \log \left(\frac{\mathrm{d}}{\mathrm{D}_{84}}\right)\right)\right. & (4=\text { LEOPOLD et al. })
\end{array}
$$

siendo, $\mathrm{V}=$ velocidad media $(\mathrm{m} / \mathrm{s}) ; \mathrm{g}=$ aceleración de la gravedad; $\mathrm{d}=$ profundidad; $\mathrm{S}=$ pendiente; $\mathrm{D}_{\mathrm{n}}=$ Diámetro del porcentaje más fino que $\mathrm{n}$.

Conviene recordar que la aplicación de estas fórmulas sólo es válida para aquellos casos en que el valor de la rugosidad aparente $\left(d / D_{n}\right)$ es superior a 3 (BRAY, 1982).

Los resultados medidos y los obtenidos teóricamente en la Rambla de Cervera permiten hacer una serie de consideraciones acerca de la bondad de las fórmulas utilizadas, el papel de la rugosidad aparente y la elección del diámetro adecuado para este tipo de trabajos. 


\section{SEGURA BELTRAN}

\subsection{Nivel de ajuste de la velocidad obtenida y la medida en el campo}

En la Tabla no 2 figuran los valores de la velocidad obtenidos a partir del $\mathrm{D}_{84}$ y del $\mathrm{D}_{90}$. En general, el nivel de ajuste es relativamente bueno, si se compara con otros estudios (BRAY, 1979, 1982; HEY, 1979). Sin embargo, los resultados son mejores para el canal principal que para el secundario.

En el canal principal los mejores ajustes se han obtenido con la fórmula de LEOPOLD et al. (31 y $26 \%$ de desviación) y con la de MANNING-LIMERINOS (38 Y 36\%). Una buena aproximación se ha conseguido también eligiendo un valor de $n$ en las tablas de CHOw (1954) de 0.05. En cambio la fórmula de KEULEGAN no parece muy adecuada. Para mejorar el ajuste de esta ecuación, HEY (1982) sugiere multiplicar por $3.5 \mathrm{el} \mathrm{D}_{84}$. Efectivamente, utilizando este coeficiente los resultados mejoran bastante, ya que se pasa de un error del 104 al $36 \%$ para el $D_{84}$.

\subsection{El papel de la profundidad del flujo en los cálculos de velocidad}

El mal ajuste obtenido en el canal secundario, con valores que superan el $100 \%$ de desviación, precisan un análisis más detallado (Tabla 2). si consideramos las variables medidas en el campo de observa que las pendientes $(0.0255$ y 0.024$)$ y el $D_{84}(50$ y $51 \mathrm{~mm}$.) son prácticamente iguales para el canal principal

\section{TABLA 2}

Velocidades calculadas a partir de Dn.

\begin{tabular}{|c|c|c|c|c|}
\hline & CANA & PRINCIPAL & CAN & ECUNDARIO \\
\hline & Velocidad & $\%$ de desviación ${ }^{1}$ & Velocidad & $\%$ de desviación \\
\hline A) A partir del $D_{84}$ & & & & \\
\hline MANNING-LIMERINOS & 1.1921 & 38 & 0.7939 & 146 \\
\hline LEOPOLD & 1.1302 & 31 & 0.7281 & 125 \\
\hline KEULEGAN & 1.7861 & 106 & 1.2655 & 293 \\
\hline KEULEGAN(3.5 D $\left.\mathrm{D}_{84}\right)$ & 1.1700 & 36 & 0.7700 & 140 \\
\hline LACEY & 0.9117 & 5,81 & 0.6933 & 115 \\
\hline Rugosidad aparente & 3,1 & & 2,0 & \\
\hline b) A partir del $D_{90}$ & & & & \\
\hline MANNING-LIMERINOS & 1.1731 & 36 & 0.7578 & 134 \\
\hline LEOPOLD & 1.0937 & 26 & 0.6914 & 115 \\
\hline KEULEGAN & 1.7668 & 104 & 1.2288 & 281 \\
\hline MANNING (0.07) & 0.6582 & 25 & 0.4925 & 53 \\
\hline MANNING (0.05) & 0.9215 & 6 & 0.6895 & 112 \\
\hline Rugosidad aparente & 2,9 & & 1,8 & \\
\hline
\end{tabular}

1.- El porcentaje de desviación se ha calculado como $\left(V_{\mathrm{c}}-\mathrm{V}_{\mathrm{o}} / \mathrm{V}_{\mathrm{o}}\right) \times 100$, siendo $\mathrm{V}_{\mathrm{e}}$, la velocidad calculada y $\mathrm{V}_{\circ}$, la velocidad observada 
y el secundario, mientras que la profundidad varia en $5 \mathrm{~cm}$. A pesar de $\mathbf{l a}$ similitud de estas cifras, la velocidad medida en el canal principal $(0.86 \mathrm{~m} / \mathrm{s})$ supera en 2.68 veces a la del canal secundario $(0.32 \mathrm{~m} / \mathrm{s})$ (Tabla 1$)$. De estos datos se podria deducir que la profundidad juega un papel fundamental en la determinación de la velocidad. La baja velocidad registrada en el canal secundario se debe a que probablemente corresponde a una velocidad típica de la "subcapa laminar"; es decir, de la zona donde la resistencia al flujo es máxima y la velocidad más baja.

De hecho, en este caso no se cumple la condición de que la rugosidad aparente $\left(d / D_{n}\right)$ sea mayor de 3 . En efecto, con $D_{84}$ este valor para el canal principal es de 3.1, mientras en el secundario es de 2.05. Para el $D_{90}$, los mismos cálculos son de 2.98 en el primer caso y de 1.87 en el segundo (Tabla 2). La escasa variación de la rugosidad aparente en el canal principal para ambos percentiles explicaria que el ajuste no varia demasiado cuando se calcula la V con $\mathrm{D}_{84}$ o con $\mathrm{D}_{90}$; en cambio en el canal secundario, con una variación significativa, los resultados mejoran bastante con el $\mathrm{D}_{90^{\circ}}$.

\subsection{El tamaño de los sedimentos}

Las fórmulas utilizadas se basan en el papel que juegan los sedimentos del lecho a la hora de frenar la velocidad del agua. Sin embargo, si se establece una relación entre los diferentes calibres -por ejemplo, $D_{84} / D_{50}$ - y entre las velocidades obtenidas con esos diámetros $\left(\mathrm{V}_{84} / \mathrm{V}_{50}\right)$ en el canal principal, los resultados no varian en la misma proporción. Así, la razón entre los diámetros es de 1.69, mientras que la misma relación entre las velocidades indica que la $V_{84}$ disminuye sólo en 0.82, utilizando la ecuación de MANNING-LIMERINOs y la de LEOPOLD et al, mientras que con la de Keulegan el descenso es de 0.87 (Tabla 3).

TABLA 3

Relaciones entre velocidades y diámetro de los sedimentos.

\begin{tabular}{lcc}
\hline & $\left(D_{84} / D_{50}\right)$ & $\left(V_{84} / V_{50}\right)$ \\
\hline MANNING-LIMERINOS & 1.69 & 0.82 \\
LEOPOLD & 1.69 & 0.82 \\
KEULEGAN & 1.69 & 0.87 \\
\hline
\end{tabular}

El análisis de estos resultados nos permite hacer una serie de observaciones sobre algunas cuestiones. En primer lugar, las fórmulas de MANNING-LIMERINOS y LEOPOLD et al. enfatizan más la rugosidad de los sedimentos del lecho que la de KEULEGAN, y por ello las velocidades obtenidas con las primeras son más bajas que con la última. Por otra parte, en estas fórmulas el calibre de los materiales está muy mediatizado por las constantes que intervienen en ellas. Por ello, los valores de velocidad calculados con distintos diámetros no presentan grandes 


\section{SEGURA BELTRAN}

diferencias entre si. En consecuencia, las diferencias existentes entre la velocidad medida y la calculada seguramente deberian ajustarse introducien do otros valores de la curva granulométrica. Bien es verdad que las fórmulas se complicarian bastante, pero seria una manera más precisa de estimar la rugosidad que ejercen los sedimentos sobre el flujo.

\section{La capa superficial de sedimentos más groseros}

En algunos rios, el calibre de los materiales de la superficie del cauce suele superar a los de la capa subyacente. Esta caracteristica sedimentológica, también presente en las ramblas, (SEGURA, 1990), recibe el nombre de armour) armor y pavement. Para algunos autores son términos sinónimos (MILHous, 1982; KLINGEMAN y EMMET, 1982; SCHICK, LEKACH y HASSAN, 1987), mientras que otros los diferencian e incluso los utilizan en sentido opuesto.

Así, PARKer, Klingeman y MClean (1982 c); ANDREws, PARKER, (1987) y SUTHERLAND (1987) establecen diferencias entre armour y pavement. El último término define la capa de materiales más gruesos cuyas partículas se mueven cuando se dan las condiciones hidrodinámicas idóneas; en cambio, la capa de armour se consideraria esencialmente inmóvil. Por el contrario, KellerHALs (1967) y BRAY y CHURCH (1980), usan el término pavement para los lechos móviles. Por nuestra parte, (SEGURA, 1990) hemos utilizado têrmino armour para definir los lechos móviles de las ramblas. Sin embargo las tendencias más recientes se inclinan por utilizar el término pavement, que es el que se usará en este trabajo para aludir a la capa superficial más grosera de las ramblas. No obstante, independientemente del vocablo, la capa grosera que forma el lecho funcional de las ramblas, es móvil.

En general los temas más investigados se refieren a los procesos de formación y a la función de esta capa. Por lo que respecta a la primera cuestión, los procesos que intervienen son ligeramente diferentes según se trate de una capa móvil o de una inmóvil. La capa de armour se formará cuando el flujo remueve una mezcla heterogénea de materiales, que tienen cortada su fuente de alimentación por cualquier causa (por ejemplo, la construcción de un embalse). En estos casos el flujo arrastra las partículas más finas, de forma que la superficie se vuelve más grosera y la ratio de transporte decrece, hasta que el movimiento de las partículas cesa por completo.

En el caso del pavement, la teoría más generalizada sugiere que esta capa se mantiene con descargas pequeñas, aunque se destruye con los caudales punta. Durante el limbo de recesión, el pavimento se vuelve a formar por la deposición primero de las partículas de mayor calibre y de los materiales más finos después (PARKer, Klingeman y MClean (1982 c). Sin embargo, ANDREws y PARKER (1987) sostienen que la textura superficial más grosera se mantiene incluso durante el momento álgido de las crecidas, ya que sólo se remueve una pequeña parte del material. 
Por otra parte, la mayoria de autores coincide en atribuir al pavimento una función de protección y control de la erosión y el transporte:

-la protección microscópica la ejercen las partículas de mayor calibre sobre los materiales más finos. Estos últimos tienen mayor movilidad intrinseca, pero en una mezcla heterogénea quedan protegidos por los materiales más gruesos. Por el contrario, la fracción más gruesa, al permanecer en superficie, queda más expuesta al flujo. Asi de forma indirecta, el pavimento controla que la ratio de transporte sea similar en ambas fracciones.

-la protección macroscópica, es la que realiza la capa superficial sobre la subcapa subyacente. De hecho, el pavimento reduce la movilidad de los materiales más finos del subpavimento.

En el caso de la rambla de Cervera, el análisis de ocho muestras recogidas en la sección transversal cuando ya había cesado la crecida, permite una discusión sobre las características de las dos capas y las relaciones establecidas entre ellas.

\subsection{Caracteristicas sedimentológicas de las dos capas}

Los parámetros granulométricos de los sedimentos quedan reflejados en la Tabla 4. En todos los casos se produce una disminución del tamaño medio -más significativo en mm. que en tamaño $\phi)$ - de las muestras de superficie (M1, M2, M3, y M4) en relación a las subsuperficiales. Ello demuestra las diferencias en la distribución de los sedimentos por tamaños Tabla 5). En el pavimento existe una alta proporción de materiales con calibre superior a $4 \phi$, mientras que en el subpaviemnto se registra un mayor porcentaje de materiales con calibres inferiores a $-1 \phi$.

TABLA 4

Parámetros estadisticos de las muestras.

\begin{tabular}{lllrl}
\hline & $M z$ & $\sigma$ & $S k$ & $K g$ \\
\hline M1(p) & -4.5 & 0.83 & -0.35 & 0.89 \\
M5(sp) & -3.6 & 1.22 & -0.16 & 0.91 \\
M2(p) & -3.6 & 0.96 & 0.05 & 1.09 \\
M6(sp) & -2.6 & 1.6 & 0.23 & 1.44 \\
M3(p) & -5.0 & 0.93 & 0.43 & 0.81 \\
M7(sp) & -2.7 & 1.30 & 0.33 & 0.88 \\
M4(p) & -4.9 & 1.13 & -0.10 & 0.65 \\
M8(sp) & -3.26 & 1.70 & 0.35 & 0.65 \\
\hline
\end{tabular}

Tanto en el pavimento como en el subpavimento, existe cierta tendencia al predominio de tamaño $4 \phi$ (16 mm.), detectado también en otros trabajos 
(SEgura, 1990). Sin embargo, en las muestras del pavimento, se produce una segunda acumulación en torno al $5.5 \phi$ (45 mm.), mientras que en el subpavimento el segundo máximo oscila entre -2 y $-3 \phi$ (4 y 8 mm) (fig. $n^{2} 2$, Tabla 5).

TABLA 5

Distribuciones por tamaños de los materiales de las muestras del pavimento (p) y del subpavimento (sp) en \%.

\begin{tabular}{|c|c|c|c|c|c|c|c|c|c|c|c|c|c|}
\hline & -6 & $-5,5$ & -5 & $-4,5$ & -4 & $-3,5$ & -3 & $-2,5$ & -2 & $-1,5$ & -1 & $<-1$ & $\phi$ \\
\hline M1 (p) & 19,8 & 24,7 & 19,2 & 3,4 & 19,0 & 8,9 & 2,1 & 1,7 & 0,9 & 0,09 & 0,05 & 0,009 & \\
\hline M5 (sp) & 5,3 & 11,05 & 3,2 & 9,3 & 20,0 & 10,2 & 16,5 & 16,0 & 1,9 & 5,0 & 2,2 & & \\
\hline M2 (p) & 0,0 & 8,2 & 5,7 & 11,7 & 20,6 & 32,0 & 8,7 & 10,0 & 2,2 & 0,3 & 0,2 & 0,1 & \\
\hline M6 (sp) & 0,0 & 0,0 & 0,0 & 3,8 & 9,3 & 26,0 & 10,3 & 14,9 & 12,3 & 2,0 & 5,8 & 15,4 & \\
\hline M3 (p) & 7,5 & 26,8 & 13,0 & 11,4 & 27,5 & 11,5 & 1,3 & 0,0 & 0,1 & & & & \\
\hline M7 (sp) & 0,0 & 0,0 & 0,0 & 0,0 & 17,5 & 24,8 & 6,7 & 11,0 & 12,2 & 2,5 & 10,1 & 14,9 & \\
\hline M4 (p) & 25,7 & 9,3 & 15,0 & 29,4 & 16,1 & 2,8 & 1,4 & 0,15 & & & & & \\
\hline M8(sp) & 0,0 & 15,5 & 0,0 & 4,5 & 23,6 & 16,8 & 4,9 & 7,1 & 8,2 & 1,9 & 7,5 & 9,6 & \\
\hline
\end{tabular}

La clasificación de los materiales del subpavimento (cuadro $\mathbf{n}^{\circ} 4$ ) empeora en todos los casos; esto es, está formado por granulometrias muy heterogéneos. La asimetria -independiente del calibre de los sedimentos-, no presenta una tendencia clara, ya que señala únicamente la forma de la distribución. Por otra parte, la kurtosis parece ser más grande en el subpavimento. Ello podría reflejar el hecho de que las acumulaciones en los tamaños predominantes alcanzan valores más elevados en el subpavimento que en el pavimento. En otras palabras, los sedimentos se concetran en pocos tamaños con porcentajes muy similares en el pavimento, mientras que en la capa subsuperficial los sedimentos se reparten de un modo más homogéneo pero en más intervalos (Tabla 5). Por último, las curvas acumuladas semilogaritmicas son más tendidas en el subpavimento que en el pavimento (figura $n^{\circ} 3$ ).

\subsection{Relaciones entre el pavimento y la capa subyacente}

Los valores (en mm.) de los diferentes diámetros $\left(\mathrm{D}_{50}, \mathrm{D}_{75}, \mathrm{D}_{84} \mathrm{y}_{90}\right)$ del pavimento $\mathrm{y}$ del subpavimento permiten avanzar algunas pautas sobre los fenómenos de pavimentación fluvial. En primer lugar, para un diámetro determinado, el calibre es superior en la capa superficial que en la subyacente. La relación entre diámetros del pavimento y del subpavimento $\left(\mathrm{D}_{\mathrm{p}} / \mathrm{D}_{\mathrm{sp}}\right)$ oscila entre 1.5 y 3.58 y la media para cada diámetro se sitúa en torno al 2.5 (Tabla 6). Estas cifras son similares a las obtenidas por otros autores en ríos o en 


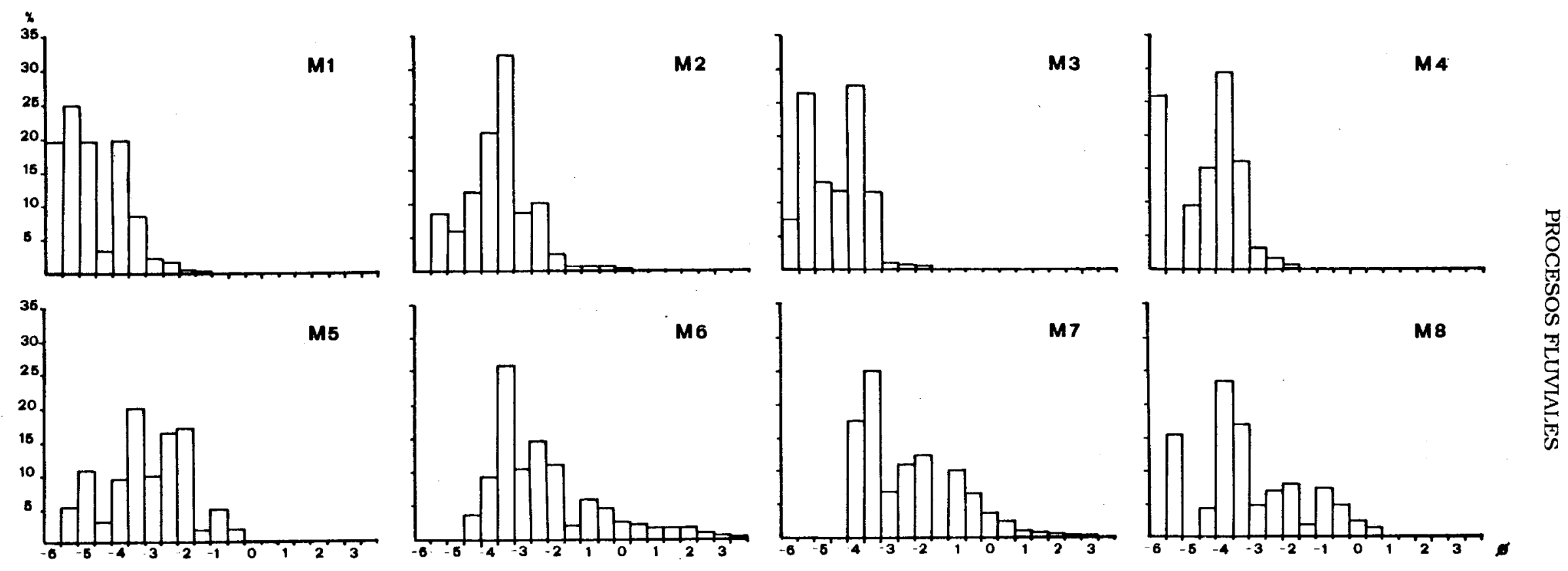

Fig. 2: Histogramas de frecuencias de las muestras del pavimento (M1, M2, M3 y M4) y del subpavimento (M5, M6, M7 y M8). 
laboratorios (Tabla 7). Asímismo, se sitúan dentro del rango indicado por ANDREWS y PARKER (1987). Estos autores señalan que generalmente la ratio $D_{\mathbf{p}} /$ $\mathrm{D}_{\mathrm{sp}}$ oscila entre 2 y 6.
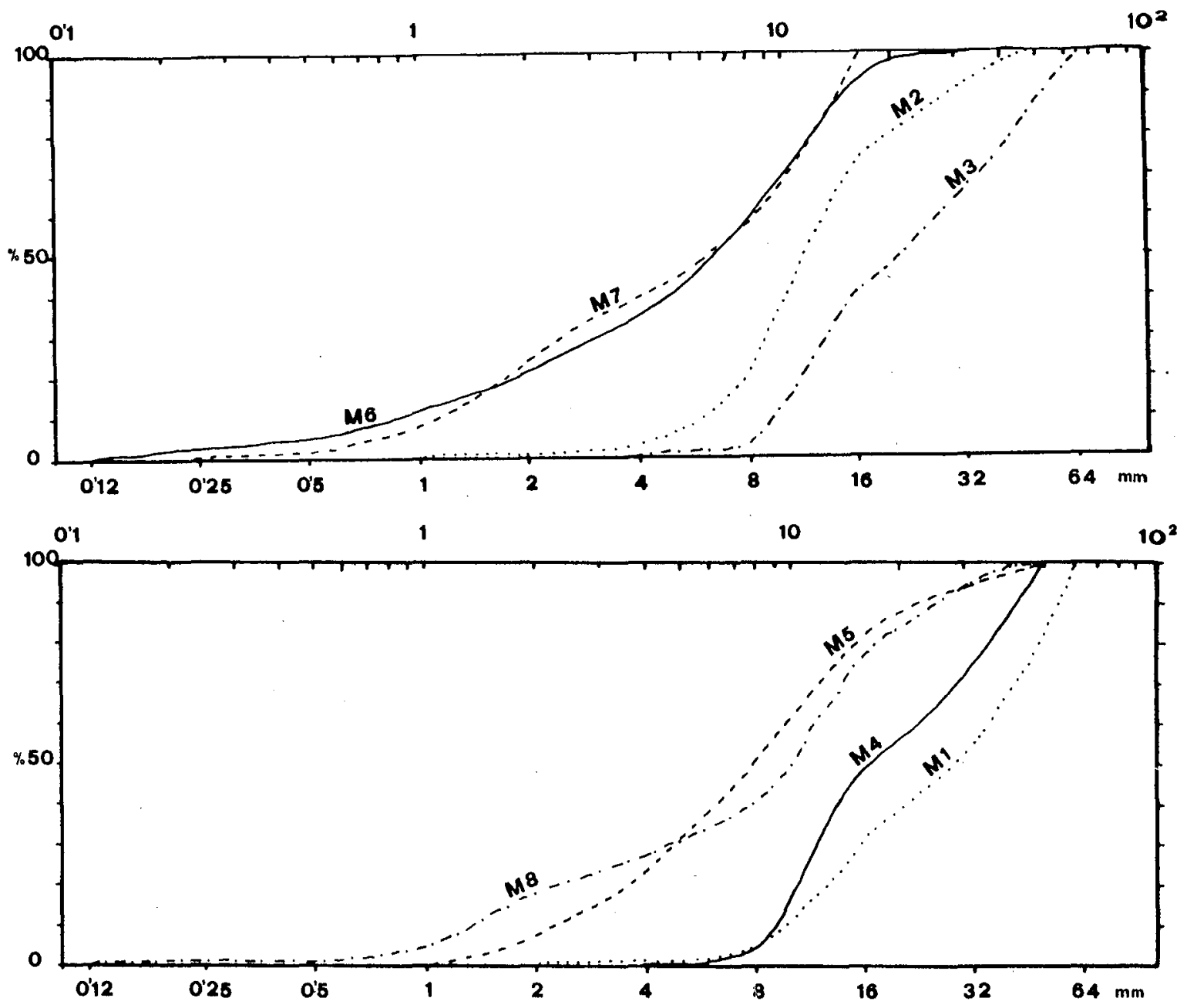

Flg. 3: Curvas acumuladas semilogaritmicas de las muestras del pavimento y del subpavimento.

Debido al escaso número de muestras no se puede deducir nada acerca de las diferencias existentes entre la ratio de los diámetros, únicamente cabe señalar que en unos casos es elevada mientras que en otros es bastante baja. Por ejemplo, la relación $\mathrm{D}_{\mathrm{p}} / \mathrm{D}_{\mathrm{sp}}$ entre M2 (pavimento) y M6 (subpavimento) alcanza un valor medio de 1.77, mientras esta misma relación para M3 y M7 se eleva a 3.5. La ubicación en el corte de estos dos grupos de muestras (figura $\mathbf{n}^{\circ}$ $1 \mathrm{C})$ difiere sensiblemente, aunque no podemos afirmar que sea la causa de la variabilidad de la ratio $\mathrm{D}_{\mathrm{p}} / \mathrm{D}_{\mathrm{sp}}$ entre $\mathrm{M} 2$ (pavimento) y M6 (subpavimeto) alcanza un valor medio de 1.77, mientras esta misma relación para M3 y M7 se eleva a 
PROCESOS FLUVIALES

TABLA 6

Relación entre el calibre de los sedimentos del pavimento y del subpavimento.

\begin{tabular}{lrrrrr}
\hline & $D_{s 0}$ & $D_{75}$ & $D_{s 4}$ & $D_{s o}$ & MEDIA \\
\hline M1(p) & 28,0 & 45,0 & 50,0 & 54,0 & \\
M5(sp) & 7,8 & 17,0 & 17,0 & 23,0 & \\
Dp/Dsp & 3,6 & 2,6 & 2,9 & 2,3 & 2,8 \\
M2(p) & 11,5 & 16,5 & 23,0 & 30,0 & \\
M6(sp) & 6,2 & 11,0 & 13,0 & 14,5 & \\
Dp/Dsp & 1,8 & 1,5 & 1,8 & 2,0 & 1,7 \\
M3(p) & 21,0 & 39,0 & 46,0 & 52,0 & \\
M7(sp) & 6,1 & 11,3 & 13,0 & 14,5 & \\
Dp/Dsp & 3,4 & 3,4 & 3,5 & 3,6 & 3,5 \\
M4(p) & 16,5 & 32,0 & 37,5 & 41,5 & \\
M8(sp) & 10,0 & 15,0 & 19,5 & 25,0 & \\
Dp/Dsp & 1,6 & 2,1 & 1,9 & 1,6 & 1,8 \\
Media Dp/Dsp & 2,6 & 2,4 & 2,5 & 2,4 & \\
\hline
\end{tabular}

3.5. La ubicación en el corte de estos dos grupos de muestras (figura $n^{\circ} 1 \mathrm{C}$ ) difiere sensiblemente, aunque no podemos afirmar que sea la causa de la variabilidad de la ratio $D_{p} / D_{s p}$. Seria interesante estudiar si estas diferencias pueden atribuirse a la disposición de las muestras sobre las microformas que se parecian en el canal.

\section{TABLA 7}

Relaciones entre $D p / D s p$ encontradas por otros investigadores.

\begin{tabular}{|c|c|c|c|c|}
\hline AUTOR & $R I O / E X P$ & $D_{s o}(p)$ & $D_{50}(s p)$ & $D_{s 0}(p) / D_{s 0}(s p)$ \\
\hline PARKER et al. (1982 a) & Dak Creek (Dregón) & $54 \mathrm{~mm}$ & $20 \mathrm{~mm}$ & 2,70 \\
\hline PARKER et al. (1982 b) & Elbow River (Canadá) & $76 \mathrm{~mm}$ & $28 \mathrm{~mm}$ & 2,71 \\
\hline PARKER et al. (1982 b) & S.A.F.H.L. ${ }^{1}$ & $3,32 \mathrm{~mm}$ & $2,02 \mathrm{~mm}$ & 1,64 \\
\hline PARKER et al. (1982 b) & T.B.H.L. ${ }^{2}$ & $4,6 \mathrm{~mm}$ & $1,95 \mathrm{~mm}$ & 2,35 \\
\hline PARKER et al. (1982 c) & Snake River (U.S.A.) ${ }^{3}$ & $54 \mathrm{~mm}$ & $27 \mathrm{~mm}$ & 2,00 \\
\hline PARKER et al. (1982 c) & Clearwater River(U.S.A.) & $72 \mathrm{~mm}$ & $18 \mathrm{~mm}$ & 4,00 \\
\hline PARKER et al. (1982 c) & Veddar River (Canadá) & $44 \mathrm{~mm}$ & $19 \mathrm{~mm}$ & 2,31 \\
\hline
\end{tabular}

1. S.A.F.H.L.- St. Anthony Falls Hydraulic Laboratory of the University of Minessota, 2, T.B.H.L.- T. Blench Hydraulic Laboratory. Unlversity of Alberta, Canadá, 3, Rios que tienen barras de arena. 


\section{Discusión y conclusiones}

Los resultados obtenidos no pueden considerarse como definitivos. De hecho sólo se ha analizado un suceso, cuando lo ideal seria disponer de una serie más larga de observaciones. Mientras tanto se pueden establecer algunos puntos:

-En primer lugar, en las ramblas existe una capa de scour, cuya potencia puede variar sustancialmente en función de la rugosidad aparente, de la cohesión de los materiales, de la naturaleza del flujo, etc. Sin embargo, el comportamiento de estas variables aún no está bien definido.

-La utilización de fórmulas teóricas basadas en el calibre de los sedimentos permite estimar la velocidad del flujo. Los mejores ajustes se obtienen con aquellas ecuaciones que enfatizan la rugosidad del lecho (LEOPOLD et al., 1956 y MANNING-LIMERINOS, 1970), lo que es bastantelógico si consideramos el grueso calibre de los sedimentos que recubren el lecho de las ramblas. Por otra parte, las variables que juegan un papel fundamental en este tipo de estimaciones son la profundidad del flujo y más concretamente, la rugosidad aparente, debido a su influencia sobre el perfil de la velocidad.

-Una capa de materiales groseros protege de la erosión al material subyacente. Ambas capas presentan características sedimentológicas bastante bien definidas. El grueso calibre y la mejor clasificación de los clastos superficiales denota que han sido transportados y depositados por un flujo de energia mayor que la capa subyacente. Por otra parte, la relación casi constante entre los diferentes diámetros del pavimento y del subpavimento, reseñada también por diferentes autores, indica que este fenómeno no depende de las características de los sedimentos sino de variables del flujo todavia no determinadas.

Sin embargo, para avanzar en estos temas se necesitará en el futuro el concurso de diferentes especialistas en temas fluviales. Tal vez las observaciones de campo y la experimentación en el laboratorio ayuden a dilucidar los complejos mecanismos que gobiernan el transporte de sedimentos en rios con materiales groseros.

Agradecimientos. La autora quiere expresar su agradecmiento al Dr. Mateu del Dpto. de Geografia de la Universidad de Valencia, así como al Dr. Marco de la E.T.S. de Ingenieros de Caminos de la Universidad Politécnica de Valencia, por la lectura critica y las sugerencias aportadas a este trabajo.

\section{Bibliografia}

ANDREWS, E.E. \& PARKER, G., 1987.- Formation of a coarse surface layer as the response to gravel mobility. In THORNE, C.R., BATHURST, J.C., HEY, R.D. (eds.): Sediment transport in gravel-bed rivers. John Wiley \& Sons, 995 pp.; cf. pp. 269-328, Chichester.

BRAY,D.I., 1979. - Estimating average velocity in gravel-bed rivers. Journal of the Hydraulics Divison, A.S.C.E., 105 (HY9) Proc. Paper 14810: 1103-1122. 


\section{PROCESOS FLUVIALES}

BRAY, D.I. and ChURCH, M., 1980.- Armored versus paved gravel beds Proc. Am. Soc. Civ. Engrs., J. Hydraul. Div., 106 (HY11): 1939-1940.

BRAY, D.I., 1982.- Flow resistence in gravel-bed rivers. In HEY, R.D., BATHURST, J.C. and THORNE, C.R., (eds.): Gravel-bed rivers, John Wiley \& Sons, pp. 109-137, Chichester.

R.D., BATHURST,J.C. and ThORNE, C.R., (eds.): Gravel-bed rivers. John Wiley \& Sons, pp. 1O9137, Chichester.

CowAN, W.L., 1954.- Estimating hydraulic roughness coefficients. Agricultural Engieneering, 37 (7): 473-475.

CHow, V.T., 1954.- Open-channel hydraulics, McGraw-Hill Book Co., 680 pp., New York.

HEY, R.D., 1979.- Flow resistance in gravel-bed rivers. Journal of the Hydraulics Division, A.S.C.E., 105 (HY4): 365-379.

HEY, R.D., 1982.- Gravel-bed rivers: form and processes. In THORNE, C.R., BATHURST, J.C., HEY, R.D. (eds.): Gravel-bed rivers. John Wiley \& Sons, pp. 5-14, Chichester.

KELLERHALS, R., 1967.- Stable channels with gravel-paved beds. Journal of the waterways and Harbors Division, A.S.C.E., 93 (WW1), Proc. Paper 5091: 63-84.

KEULEGAN, G.H., 1938. - Laws of turbulent flows in open channels. Journal of Research of the United States National Bureau of Standards, 21, Research Paper Rp 1151: pp. 707-741.

KLINGEMAN, P. C., EMMETT, W.W., 1982.- Gravel bedload transport processes. In HEY, R.D., BATHURST, J.C. and THORNE, C.R., (eds.): Gravel-bed rivers. John Wiley \& sons, pp. 141180 , Chichester.

LACEY, G., 1946-47.- A theory of flow in alluvium. Journal of the Institution of Civil Engineers, 27, Paper $\mathrm{n}^{2}$ 5518: 16-47.

LEOPOLD, L.B., Wolman, M.G., and Miller, J.F., 1964.- Fluvial processes in Geomorphology. Freeman and Company, 522 pp., Sna Francisco.

LIMERINOS, J.T., 1970.- Determination of the Manning coefficient from measured bed roughness in natural channels. Water Supply Paper 1898-B, United States Geological Survey, 47 pp., Washington D.C.

Milhous, R.T., 1982.- Effect of sediment transport and flow regulation on the ecology of gravel-bed rivers. In THORNE, C.R., BATHURST, J.C., HEY, R.D. (eds.): Gravel-bed rivers. John Wiley \& Sons, pp. 819-842, Chichester.

PARKER, G. and KLINGEMAN, P.C., 1982 a .- On why gravel bed streams are paved. Water Resources Research, 18 (5): 1409-1423.

Parker, G. Dhamotharan, S. and STEFan, H., 1982 b.- Model experiments on mobile, paved gravel bed streams. Water Resources Research, 18 (5): 1395-1408.

PARKER, G., Klingeman, P.C. and McLEAN, D.G., 1982 c.- Bedload and size distribution in paved gravel-bed streams. Journal of the Hydraulics Division, A.S.C.E., 108 (HY4): 546571.

REID, I., FROSTICK, L.E. and LAYMAN,J., 1985.- The incidence and nature of bedload transport during flood flows in coarse-grained alluvial channels. Earth Surface Processes and Landforms, 10: 33-44.

Sanjaume, E., Segura, F.S., Pardo, J., López, M.J., Burguet, I., Sapiña, F., in fieri.- La sedimentación en la albufera de Valencia. Proyecto de investigación del I.V.E.I., inédito.

SEguRA, F.S., 1984.- Las terrazas de la Rambla de Cervera. Cuadernos de Geografia, 43: 129.

SEGURA BELTRÁN, F.S., 1986.- La Rambla de Cervera: some aspects concerning the sedimentology, hydrology and geomorphology. Excursion Guide-Book, C.O.M.T.A.G. Symposium, 88-103.

SEGURA, F.S., 1990.- Las ramblas valencianas. Universitat de Valencia, 229 pp., Valencia. 


\section{SEGURA BELTRAN}

SCHICK, A.P., LeKACH, J. and HASSAN, M.A., 1987.- Bed load transport in desert floods: observations in the Negev. In ThORNE, C.R., BATHURST, J.C., HEY, R.D. (eds.): Sediment transport in gravel-bed rivers. John Wiley \& Sons, pp. 617-642, Chichester.

SUTHERLAND, A.J., 1987.- Static armour layers by selective erosion. In THORNE, C.R., BATHURST, J.C., HEY, R.D. (eds.): Sediment transport in gravel-bed rivers. John Wiley \& Sons, pp. 243-267, Chichester. 